\title{
Singularity in a (2+1)-dimensional AdS scalar black hole
}

\author{
Lior M. Burko \\ Theoretical Astrophysics, California Institute of Technology, Pasadena, California 91125
}

(Received 17 August 2000; published 15 November 2000)

\begin{abstract}
We study the spacetime singularity in a (2+1)-dimensional AdS scalar black hole with circular symmetry using a quasihomogeneous model. We show that this is a spacelike, scalar curvature, deformationally strong singularity.

PACS number(s): 04.20.Dw, 04.60.Kz, 04.70.Bw
\end{abstract}

Recently there has been growing interest in asymptotically anti-de Sitter (AdS) black holes, motivated by the discovery of the Bañados-Teitelboim-Zanelli (BTZ) black hole [1] in general relativity (for reviews see $[2,3]$ ) and by the so-called AdS-CFT (conformal field theory) speculation in string theory [4]. Very recently, Pretorius and Choptuik presented results of fully nonlinear numerical simulations of the gravitational collapse of a massless, minimally coupled scalar field in $(2+1)$-dimensional [ $(2+1) \mathrm{D}]$, axially-symmetric, $\mathrm{AdS}_{3}$ spactime in classical general relativity [5]. Pretorius and Choptuik studied the critical behavior at the threshold of black-hole formation in this model, and found a continuous self-similar solution and type-II behavior with a mass-scaling law with a critical exponent $\sim 1.2$. This work was followed by Garfinkle [6], who was able to find analytically an exact solution for this model, in agreement with the numerical results of Ref. [5]. Husain and Olivier studied the same model using a different numerical approach and a different evaluation approach for the critical exponent, and reported on a mass-scaling law with a critical exponent $\sim 1.6$ [7]. This apparent discrepancy of the two estimates for the critical exponent is of great interest.

Pretorius and Choptuik also studied in Ref. [5] the nature of the singularity in their model for supercritical evolutions. They report on a scalar curvature singularity, which is spacelike and deformationally strong [8,9]. However, the evidence brought in Ref. [5] appears to be inconclusive. Specifically, Pretorius and Choptuik argue that the singularity is spacelike because the hypersurface along which the metric variables start growing unboundedly in the normal direction, and consequently the curvature invariants begin to diverge, is spacelike. Although this is consistent with the singularity being spacelike, it is only a necessary condition for it. The mass inflation [10-13] null singularity is a counterexample, where hypersurfaces of constant large (albeit finite) curvature are spacelike, although the singularity itself is null. The evidence that Pretorius and Choptuik bring for the strength of the singularity is that it is central, namely, that the proper circumference vanishes approaching the singularity. This criterion appears indeed to be sufficient evidence in spherical symmetry under very broad conditions [14], but this has never been shown in $(2+1) \mathrm{D}$ and circular symmetry.

In this paper we show, within a simplified model, that indeed this singularity is spacelike, scalar curvature, and deformationally strong. Specifically, we assume that spacetime asymptotically close to the singularity can be described by a quasihomogeneous model, i.e., we assume that spatial gradi- ents (of the metric functions and the scalar field) are much smaller than temporal gradients. This assumption seems to be justified by the results of Ref. [5], where Figs. 18 and 19 imply only mild gradients tangent to the singularity, and much steeper gradients normal to it. We shall study the solution to the Einstein-scalar equations in $(2+1) \mathrm{D}$ and circular symmetry only asymptotically close to the singularity. We thus write the metric as

$$
d s^{2}=N(r) d t^{2}-L(r) d r^{2}+r^{2} d \theta^{2}
$$

where $0 \leqslant \theta \leqslant 2 \pi$ is possibly periodic, and where $N(r)$ and $L(r)$ are non-negative functions (such that $r=$ const hypersurfaces are spacelike by construction). The coordinate $r$ is defined such that the proper circumference of circles of radius $r$ is $2 \pi r$. Note, that $r$ is a timelike coordinate. We study the solution to the Einstein-scalar equations

$$
G_{\mu \nu}+\Lambda g_{\mu \nu}=\kappa T_{\mu \nu},
$$

where $\Lambda<0$ is the cosmological constant and $T_{\mu \nu}$ $=\nabla_{\mu} \phi \nabla_{\nu} \phi-\frac{1}{2} g_{\mu \nu} \nabla^{\alpha} \phi \nabla_{\alpha} \phi$ is the energy-momentum tensor of the scalar field $\phi$. Following the convention of Ref. [5] we set the coupling constant $\kappa=4 \pi$. The $t-t$ and $r-r$ components of the field equations (2) are, correspondingly,

$$
L^{\prime}+2 r L^{2} \Lambda=4 \pi r L \phi^{\prime 2}
$$

and

$$
N^{\prime}-2 r L N \Lambda=4 \pi r N \phi^{\prime 2}
$$

These equations are coupled to the Klein-Gordon equation for the scalar field, $\square \phi=0$, whose first integral is given by

$$
\phi^{\prime 2}=\frac{L}{N} \frac{d^{2}}{r^{2}} .
$$

Here, $d^{2}$ is an integration constant, and a prime denotes differentiation with respect to $r$. (Recall that we neglect all derivatives with respect to $t$.) The $\theta-\theta$ component of the field equations is redundant, and we use it as a consistency check for our solution. This equation is

$$
L N^{\prime 2}+N N^{\prime} L^{\prime}-2 N^{\prime \prime} N L+4 \Lambda N^{2} L^{2}=8 \pi N^{2} L \phi^{\prime 2} .
$$

We next eliminate $\phi$ from Eqs. (3) and (4) using Eq. (5), and find that 


$$
L^{\prime}+2 r L^{2} \Lambda=4 \pi d^{2} \frac{L^{2}}{N r}
$$

and

$$
N^{\prime}-2 r L N \Lambda=4 \pi d^{2} \frac{L}{r} .
$$

Next, we present a simple solution to Eqs. (7) and (8), which is a generic solution. Then, we show that, in fact, this solution is the only generic solution. Following the analyses of Refs. $[15,16]$ we seek a solution for which asymptotically close to the singularity the metric functions behave like $L(r)=A r^{\alpha}$ and $N(r)=B r^{\beta}$, where $A, B>0$ are constants. (At larger distances from the singularity higher-order terms in $r$ become important.) We next seek a solution to Eqs. (7) and (8), and demonstrate its validity by requiring consistency with the fully-nonlinear (and inhomogeneous) numerical simulations of Ref. [5]. Substituting this ansatz we find first that $\alpha, \beta>0$, and that asymptotically close to $r=0$ the $\Lambda$-coupled terms are negligible. (This was also found in Ref. [6].) Next, we find that the solution imposes on $\alpha$ and $\beta$ a relation. Specifically, we find that $\alpha=\beta$. Also, we find that $B=4 \pi d^{2} A \alpha^{-1}$. Note, that we assume here $\alpha \neq 0$. The scalar field satisfies asymptotically close to the singularity $\phi^{\prime 2}$ $=\alpha /\left(4 \pi r^{2}\right)$. (The case where $\alpha=0$ corresponds to a vanishing scalar field, and corresponds to the vacuum BTZ solution, for the case for which $\theta$ is periodic.) The dependence of the solution on $\Lambda$ enters only at higher-order terms $O\left[r^{2(\alpha+2)}\right]$. Also, Eq. (6) is satisfied by our solution at this order. (When higher-order terms in $r$ are considered each order will decrease the error associated with the truncated solution of the lower-order solution.) In fact, one does not have to assume the ansatz that $N(r)$ and $L(r)$ are given by simple powers of $r$. Instead, if one assumes that the $\Lambda$-coupled terms in Eqs. (7) and (8) are negligible (close to $r=0)$, one can solve these equations readily, and obtain that $L(r)=A r^{\alpha}$ and $N(r)=4 \pi d^{2} A \alpha^{-1} r^{\alpha}$ as an exact and unique solution (with no $\Lambda$ term). Our solution here is generic in the sense that it relies on the right number of freely-specifiable parameters [recall that we have the freedom to rescale the $t$ coordinate, namely $t \rightarrow \tilde{t}=T(t)$, such that $d$ can be set equal to unity without loss of generality].

We next show, following Nolan, that this generic solution is the only generic solution [17]. Defining $X=L N$ and $Y$ $=N / L$, Eqs. (7) and (8) become

$$
\begin{aligned}
& X^{\prime}=8 \pi \frac{d^{2}}{r} X Y^{-1}, \\
& Y^{\prime}=4 \Lambda r X^{1 / 2} Y^{1 / 2} .
\end{aligned}
$$

Next, we define $u=Y^{1 / 2}$, and obtain the second order equation

$$
r u^{\prime \prime}=\left(u-4 \pi \frac{d^{2}}{u}\right)^{\prime}
$$

whose first integral is

$$
r u^{\prime}=2 u-4 \pi \frac{d^{2}}{u}+4 k
$$

where $4 k$ is an arbitrary constant. Equation (12) can be separated and written as

$$
\int \frac{u}{u^{2}+2 k u-2 \pi d^{2}} d u=2 \int \frac{d r}{r} .
$$

Factorizing the denominator on the left hand side and using undetermined coefficients gives

$$
\frac{u}{u^{2}+2 k u-2 \pi d^{2}}=\frac{\beta+k}{2 \beta} \frac{1}{u+k+\beta}+\frac{\beta-k}{2 \beta} \frac{1}{u+k-\beta},
$$

which integrates to give logarithmic terms. Then taking the exponential of both sides gives

$$
|u+k+\beta|^{(\beta+k) / 2 \beta}|u+k-\beta|^{(\beta-k) / 2 \beta}=e^{c} r^{2},
$$

where $c$ is an arbitrary constant and $\beta^{2}=k^{2}+2 \pi d^{2}$. Notice that $\beta \pm k>0$ (we rule out $d=0$ in which case the scalar field is absent; also $\beta$ means the positive root of $\beta^{2}$ ). Equation (13) determines $Y(r)$ implicitly and Eqs. (10) and (12) can be used to give $X$ in terms of $u$ (or $Y$ ). Specifically, we find that

$$
2 \Lambda r^{2} X^{1 / 2}=2 u-4 \pi \frac{d^{2}}{u}+4 k
$$

We determine the asymptotic behavior of $X, Y$ (and hence $N$ and $L)$ as $r \rightarrow 0$ as follows. Note that $u \geqslant 0$. The left hand side of Eq. (13) must vanish at $r=0$. Since $u+k+\beta>u$ $+k-\beta$, we infer that

$$
\lim _{r \rightarrow 0} u(r)=\beta-k>0 .
$$

Thus we can write

$$
u(r)=\beta-k+\epsilon(r)
$$

where

$$
\epsilon(r)=o(1), \quad r \rightarrow 0 .
$$

Here, $o$ is defined such that $f(x)=o[g(x)]$ as $x \rightarrow 0$ implies that $f / g \rightarrow 0$ as $x \rightarrow 0$. Substituting this into Eq. (13) yields

$$
\epsilon(r)=\epsilon_{0} r^{4 \beta /(\beta-k)}+\epsilon_{1}(r),
$$

where $\epsilon_{1}=o(\epsilon)$ as $r \rightarrow 0$ and $\epsilon_{0}$ is a constant which is determined by $\beta, k$, and $c$. Equations (15) and (16) are sufficient to determine the leading order behavior of $X$ via Eq. (14), i.e.,

$$
X^{1 / 2}=\frac{2 \beta}{\Lambda(\beta-k)} \epsilon_{0} r^{2[(\beta+k) /(\beta-k)]}+o(\epsilon) .
$$

Then the original metric functions $N, L$ satisfy 


$$
\begin{aligned}
& N=u X^{1 / 2} \sim \frac{2 \beta}{\Lambda} \epsilon_{0} r^{2[(\beta+k) /(\beta-k)]}, \quad r \rightarrow 0, \\
& L=\frac{X^{1 / 2}}{u} \sim \frac{2 \beta}{\Lambda(\beta-k)^{2}} \epsilon_{0} r^{2[(\beta+k) /(\beta-k)]}, \quad r \rightarrow 0 .
\end{aligned}
$$

This proves that in general, the metric functions $N, L$ of these space-times display power-law behavior as the singularity is approached, with the same power of $r$ in each function. [The $\Lambda^{-1}$ in Eq. (17) and Eq. (18) can be absorbed into $\epsilon_{0}$ by rescaling the $t$ coordinate.] Also, we find that $\alpha=2(\beta$ $+k) /(\beta-k)$.

With this solution we first show that the singularity is scalar polynomial. Note that all the following expressions are given to leading order in $r$. Specifically, we find the Kretschmann scalar $R_{\mu \nu \rho \sigma} R^{\mu \nu \rho \sigma}=3 \alpha^{2} /\left(A^{2} r^{2 \alpha+4}\right)$, and the Ricci scalar $R=\alpha /\left(A r^{\alpha+2}\right)$. Both curvature scalars diverge approaching $r=0$. Next, we show that this singularity is strong in the sense of Tipler [8] (or deformationally strong in the sense of Ori [9]). The timelike-timelike component of the Ricci tensor in a parallel-propagated frame is given by $R_{(0)(0)}=\alpha /\left(A r^{\alpha+2}\right)$. Reexpressing that in terms of the proper time of a radial observer (who follows a $t=\mathrm{const}$ timelike geodesic), we find that

$$
R_{(0)(0)}(\tau)=4 \frac{\alpha}{(\alpha+2)^{2}} \frac{1}{\tau^{2}},
$$

where $\tau$ is (future directed) proper time, set such that $\tau$ $\rightarrow 0^{-}$approaching the singularity. Here, and in what follows, we find $R_{(0)(0)}(\tau)$ to leading order in $\tau^{-1}$. [The geodesic equation for this geodesic is $\ddot{r}+(\alpha / 2 r) \dot{r}^{2}=0$, whose solution is $\tau(r)=-2 A^{1 / 2}(\alpha+2)^{-1} r^{(\alpha+2) / 2}$, where an overdot denotes differentiation with respect to $\tau$.] Note that $R_{(0)(0)}(\tau)$ is independent of $A$. [Also $R_{\mu \nu \rho \sigma} R^{\mu \nu \rho \sigma}(\tau)=48 \alpha^{2}(\alpha$ $+2)^{-4} \tau^{-4}$ is independent of $A$.] This is similar to the independence of $R_{(0)(0)}(\tau)$ of the density (or pressure) in the Friedmann-Robertson-Walker cosmology near the singularity, or the independence of the Kretschmann scalar of the mass in Schwarzschild, or of the density in the FriedmannRobertson-Walker cosmology [18].

We next use a theorem by Clarke and Królak, according to which a sufficient condition for the singularity to be strong in the sense of Tipler is that

$$
\int^{\tau} d \tau^{\prime} \int^{\tau^{\prime}} d \tau^{\prime \prime} R_{(0)(0)}\left(\tau^{\prime \prime}\right)
$$

diverges as $\tau \rightarrow 0^{-}$[19]. From Eq. (19) it is clear that this is indeed the case, as this quantity diverges logarithmically in $\tau$ as $\tau \longrightarrow 0^{-}$, such that we show that the singularity is indeed deformationally strong. (Note that the proof of the ClarkeKrólak theorem can be easily extended to $2+1$ dimensions.)

Finally, we make the following remarks on the applicability of our homogeneous model. First, we find that asymptotically close to the singularity the scalar field diverges like

$$
\phi(r)=\left(\frac{\alpha}{4 \pi}\right)^{1 / 2} \ln r .
$$

Indeed, the fully-nonlinear and inhomogeneous simulations find this logarithmic divergence for $\phi(r, t)$ [20]. This, however, provides us also with a quantitative check for the predictions of the homogeneous model. Specifically, the amplitude $(\alpha / 4 \pi)^{1 / 2}$ of the scalar field involves the same parameter $\alpha$ as in the metric functions $N, L$. Consequently, our homogeneous model captures the pointwise behavior at the singularity well if fully-nonlinear and inhomogeneous simulations confirm our prediction that

$$
\left(\frac{r \partial L(r, t) / \partial r}{4 \pi L(r, t)}\right)^{-1 / 2} \phi(r, t) \frac{1}{\ln r} \rightarrow 1
$$

as $r \rightarrow 0$ towards any point along the singularity. Second, the numerical simulations also indicate that the Ricci scalar grows approaching the singularity like $R(\tau) \approx \tau^{-2}$ [20]. Indeed, in our model we find that $R(\tau)=4 \alpha(\alpha+2)^{-2} \tau^{-2}$. In addition to the right behavior as a function of proper time, we also predict a specific dependence on $\alpha$, which can be checked numerically. Finally, the quasihomogeneity we assume is supported by the simulations in Ref. [5], where only mild (possibly oscillatory) dependence on $t$ is reported.

We showed that in a quasihomogeneous model for the singularity in $(2+1) \mathrm{D}$ AdS black hole with a self-gravitating scalar field in circular symmetry, the singularity is spacelike, scalar curvature, and deformationally strong. We believe that this simple model captures the pointwise behavior near the singularity when one allows for inhomogeneities. The question of how the solution is modified by inclusion of inhomogeneities or angular momentum remains open.

I am indebted to Matt Choptuik, Brien Nolan, and Frans Pretorius for useful discussions. This research was supported by NSF Grant No. AST-9731698.
[1] M. Bañados, C. Teitelboim, and J. Zanelli, Phys. Rev. Lett. 69, 1849 (1992).

[2] S. Carlip, Class. Quantum Grav. 12, 2853 (1995).

[3] R. B. Mann, in Internal Structure of Black Holes and Spacetime Singularities, Vol. XIII of the Annals of the Israel Physical Society, edited by L. M. Burko and A. Ori (IOP, Bristol,
1997), gr-qc/9709039.

[4] J. M. Maldacena, Adv. Theor. Math. Phys. 2, 231 (1998).

[5] F. Pretorius and M. W. Choptuik, gr-qc/0007008.

[6] D. Garfinkle, gr-qc/0008023.

[7] V. Husain and M. Olivier, gr-qc/0008060.

[8] F. J. Tipler, Phys. Lett. 64A, 8 (1977). 
[9] A. Ori, Phys. Rev. D 61, 064016 (2000).

[10] E. Poisson and W. Israel, Phys. Rev. D 41, 1796 (1990).

[11] A. Ori, Phys. Rev. Lett. 67, 789 (1991).

[12] L. M. Burko, Phys. Rev. Lett. 79, 4958 (1997).

[13] L. M. Burko and A. Ori, in Internal Structure of Black Holes and Spacetime Singularities [3].

[14] B. C. Nolan, Phys. Rev. D 62, 044015 (2000).
[15] L. M. Burko, Phys. Rev. D 58, 084013 (1998).

[16] L. M. Burko, Phys. Rev. D 59, 024011 (1999).

[17] B. C. Nolan (private communication).

[18] L. M. Burko, in Internal Structure of Black Holes and Spacetime Singularities [3], gr-qc/9711012.

[19] C. J. S. Clarke and A. Królak, J. Geom. Phys. 2, 127 (1985).

[20] F. Pretorius (private communication). 